\title{
HIGH-RESOLUTION COMPUTERISED TOMOGRAPHY IN CLINICAL ORTHOPAEDICS
}

\author{
N. D. REIS, C. ZINMAN, M. I. B. BESSER, L. Z. SHIFRIN, Y. FOLMAN, S. TOREM, \\ D. FROINDLICH, H. ZAKLAD \\ From the Departments of Orthopaedics and General Surgery, Rambam Medical Centre \\ and the Advanced Technology Centre, Haifa
}

\begin{abstract}
High-resolution computerised tomographic (CT) scanning reveals fine details of bone structure, thereby overcoming the disadvantages of the conventional CT scanner in orthopaedic diagnosis.

A number of selected cases are presented in order to help define the indications for the use of this new diagnostic tool, and an unusual case of osteoid osteoma in a vertebral body is reported.
\end{abstract}

The use of a CT scanner as a diagnostic tool in orthopaedics has been reported (Hammerschlag, Wolpert and Carter 1976; Berger and Kuhn 1978; deSantos et al. 1978; Lee, Kazam and Newman 1978; O'Connor and Cohen 1978; Wilson et al. 1978; Hermann and Rose 1979). These workers noted that the CT scans available to them were useful in delineating the extent and site of a lesion, but the method had a limited application in diagnosis since subtle changes in bone structure were not demonstrated (Hermann and Rose 1979). deSantos et al. (1978) found that conventional radiographs were far more informative in bone tumours because they demonstrated the fine details of bone architecture. To overcome this shortcoming the resolving power of the CT scan needs to be improved by at least a factor of two.

We are now using a CT scanner which is capable of high resolution. This scanner can generate a preliminary planar view which allows the accurate planning of the

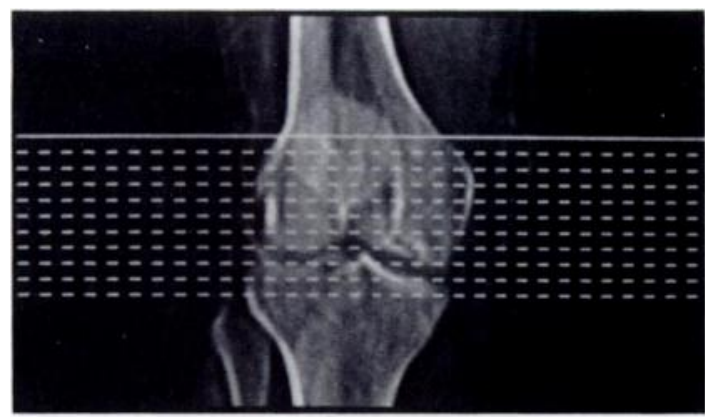

Fig. 1

Planar view of the knee used in planning the scanning session: the site, number and distance between the "slices" are determined in relation to the dotted lines. scanning session (Fig. 1). The main advantage of the CT scan is the ability to view horizontal or transverse slices, which adds greatly to conventional radiographic techniques. The fine details of bone structure are not normally visualised, except in the high-resolution CT scanner. This gives the advantages that the fine structure of the internal architecture is visualised as well as the outline of the cortex (Fig. 2); small differences in bone density are demonstrated (Fig. 3); and disturbances in the space between adjoining surfaces are visualised (Fig. 4). The precise location of the scanned slice is shown on the planar view to an accuracy of under one millimetre (Fig. 1). These capabilities provide improved diagnostic possibilities in orthopaedic surgery. This is illustrated in a number of patients, in whom additional information was obtained by the use of this technique.

\section{CASE REPORTS}

Case 1. Loose body in the hip joint. A man aged 28 had suffered pain and limitation of movement in both hips for 10 years with no change in the last five years. No other joints were involved, serology was negative for rheumatoid factor and the erythrocyte sedimentation rate was 15 millimetres in the first hour. Radiographs showed slight narrowing of both joints spaces, mild irregularity of the joint surface of the femoral heads, and some juxta-articular erosions. The diagnosis was bilateral inflammatory disease which had been inactive for several years. The patient returned to the clinic complaining of attacks of acute pain in the left hip for one month. He was unable to bear weight without crutches and movements of the left hip were severely restricted when the pain was acute; at other times movement was relatively free. The acute pain came and went without any pattern, and laboratory and radiographic examinations revealed no changes. A high-resolution CT scan of the hip (Fig. 5) showed an ossified loose
N. D. Reis, FRCS

C. Zinman, MD

M. I. B. Besser, FRCS

L. Z. Shifrin, MD

Y. Folman, MD

S. Torem, MD

D. Froindlich, $\mathrm{PhD}\}$

H. Zaklad, DEng

Requests for reprints should be sent to Dr N. D. Reis.

(1) 1982 British Editorial Society of Bone and Joint Surgery 0301-620X/82/1016-0020\$2.00
Department of Orthopaedics 'B'

Department of General Surgery 'B'

Rambam Medical Centre, Technion Faculty of Medicine, Haifa, Israel 


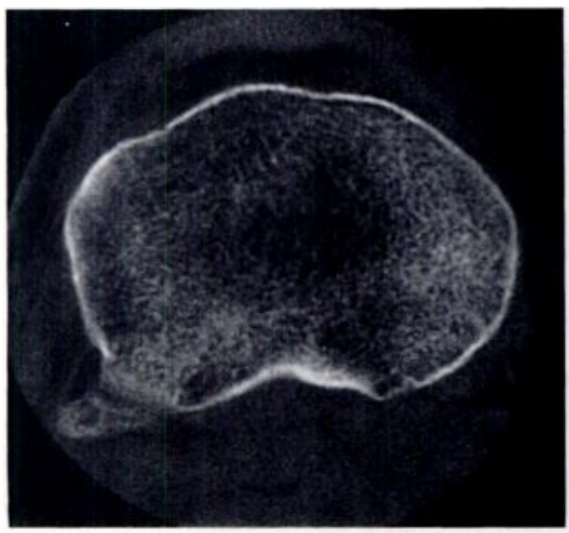

Fig. 2

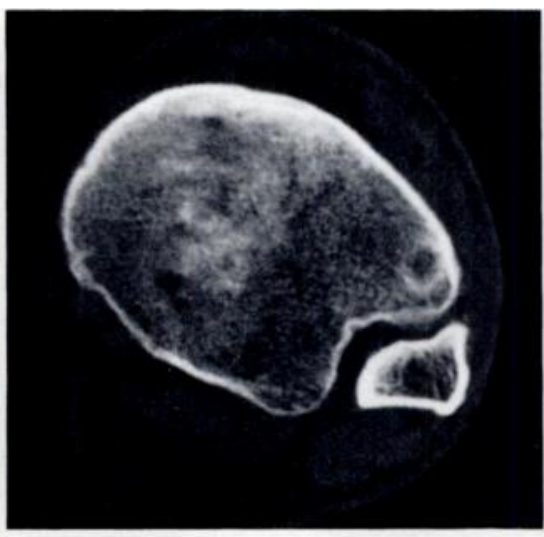

Fig. 3

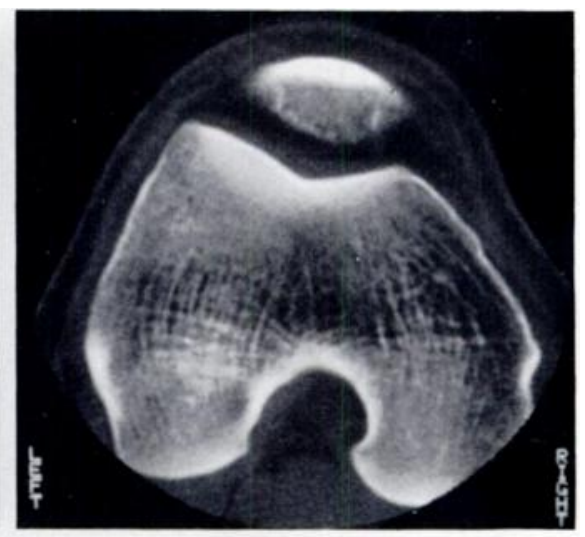

Fig. 4

Figure 2-The fine structure of the cancellous bone and the cortex of the upper tibia are clearly visualised. Figure 3-The tibia just above the ankle joint shows subchondral degenerative cystic change. Small differences in bone density are clearly seen. Figure 4-Patellofemoral joint. The subchondral bony architecture of the patella is disturbed.

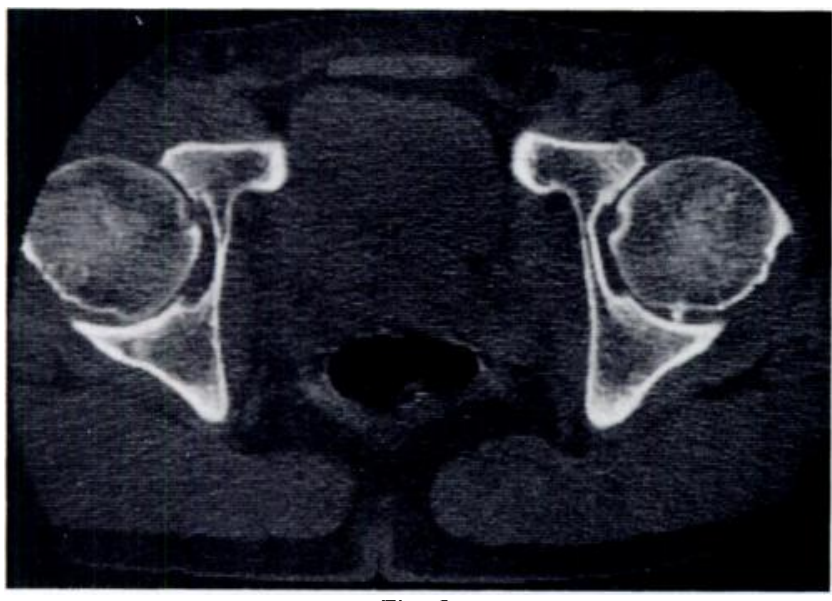

Fig. 5

Case 1. A small loose body is seen in the back of the left hip.

body about three millimetres in diameter in the posterior joint space of the left hip and confirmed the irregularity of the joint surfaces of the femoral heads. In this patient a loose body was suspected from the history and clinical examination but could be demonstrated only by high-resolution CT scan.

Case 2. Osteochondritis dissecans of the talus. A healthy man aged 22 suffered pain in his right ankle for two years when walking or running. Clinical examination was normal and radiographs showed a lesion of the upper medial pole of the dome of the talus, which was confirmed by tomography. A high-resolution CT scan (Fig. 6) gave a precise picture of the fragmented lesion in the posterosuperior talus, demonstrating accurately both its position and extent. Malleolar osteotomy was indicated to achieve a satisfactory surgical exposure.

Case 3. The talocalcaneal joint and the inferior tibiofibular syndesmosis. A man aged 20 complained of pain in the right ankle when walking on rough ground, which had persisted for a year. There was a history of injury to the ankle and swelling but no diagnosis had been made. Pain was felt mainly below the lateral malleolus, and there was only vague tenderness on the lateral side of the ankle. Radiographs of the ankle and foot were normal. Symptoms indicated that the source of pain could have been the subtalar joint, but a high-resolution CT scan of this joint showed no abnormality (Fig. 7). However, the scan of the inferior tibiofibular syndesmosis (Fig. 8) revealed post-traumatic changes with irregularity of the joint surfaces and striking anterior osteophyte formation. The tibiofibular syndesmosis can only be demonstrated by the visualisation of a horizontal slice and only a high-resolution scan can give a finely detailed picture of the bone structure.

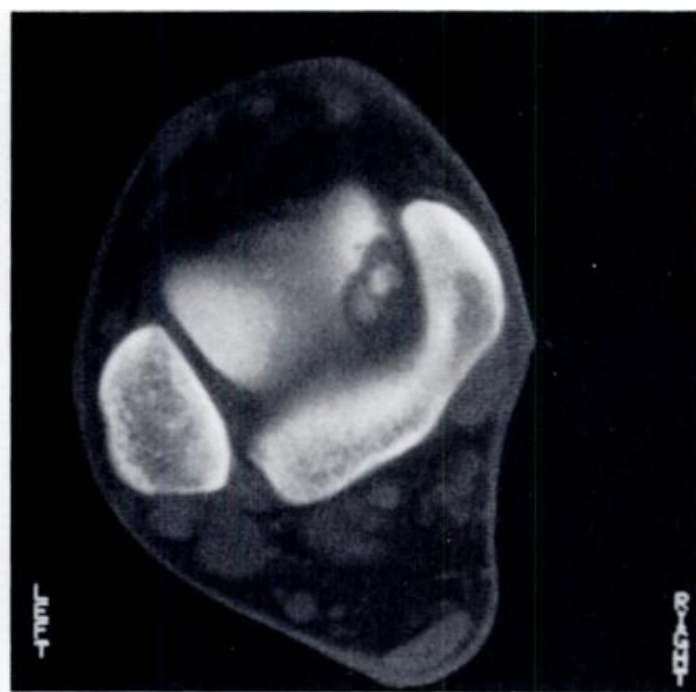

Fig. 6

Case 2. Osteochondritis dissecans of the talus. The position, extent and structure of the lesion are clearly demonstrated.

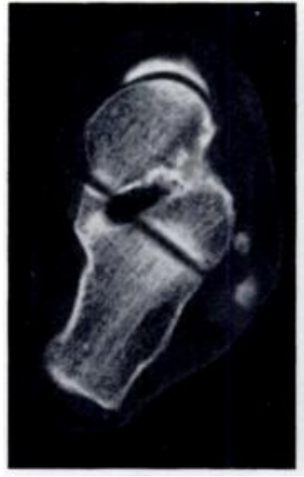

Fig. 7

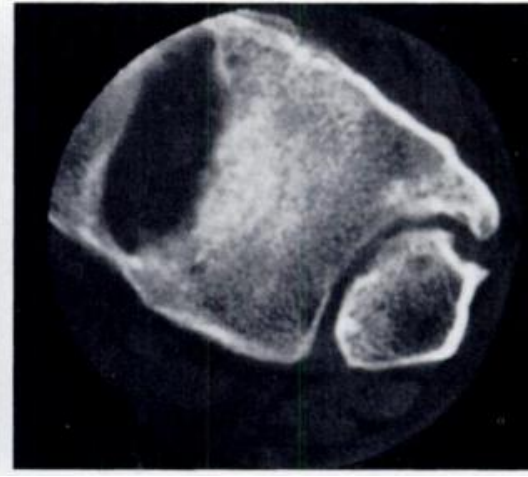

Fig. 8
Case 3. Figure 7-Normal talocalcaneal joint. Figure 8Degenerative changes and an osteophyte are shown in the inferior tibiofibular joint. The black area in the tibia is the joint space of the ankle.

Case 4. Fracture position in a plaster cast. A man aged 25 suffered a fracture of the medial malleolus extending above the level of the tibial joint surface. Reduction and screw fixation were performed, and the precision of the reduction in the horizontal plane was checked by a 


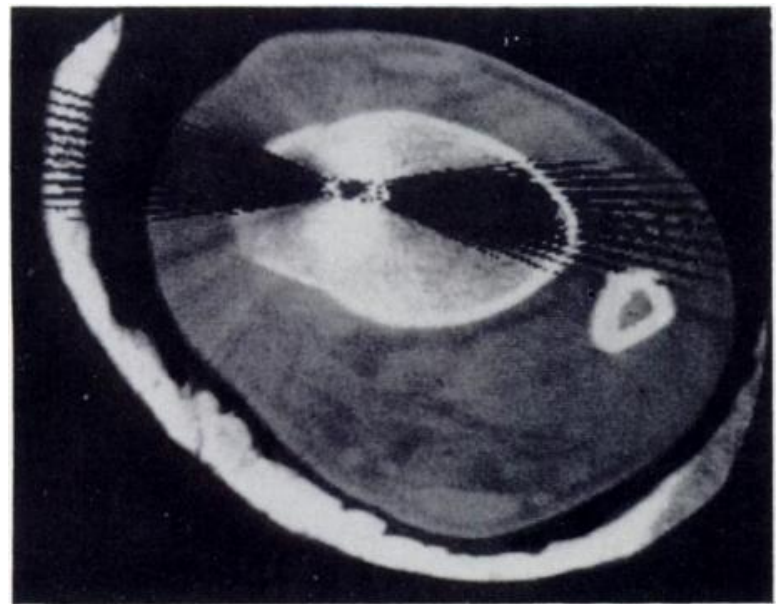

Fig. 9

Case 4. A vertical fracture of the medial malleolus has been fixed with a screw. The reduction is almost perfect; only a linear flaw in the continuity of the anterior cortex is seen. The metal screw causes a "sun-ray" effect but the plaster slab does not impair the quality of the picture.

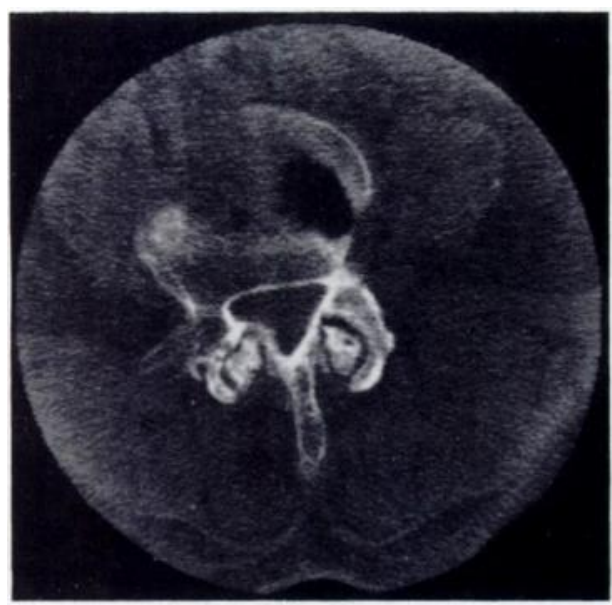

Fig. 10 high-resolution CT scan (Fig. 9). The reduction was seen to be exact and the fragment was tightly applied to the tibia. The metallic screw gave a "sun-ray" effect, but the posterior plaster slab did not interfere with the quality of the fine detail of the picture as would have been the case in a conventional radiograph. This horizontal CT slice is a useful additional check on a fracture, which may be invaluable when a plaster cannot be removed, allowing the visualisation of fine details of bone within a cast. The artefact caused by the metallic screw can if necessary be removed by use of a metal correction programme.

Case 5. Spinal stenosis and posterior joint disease of the spine. A woman aged 58 had backache for many years and for two years had suffered increasing pain in the buttocks and legs on walking, until she was able to walk only 30 yards before having to rest. On examination movement in her lumbar spine was slightly reduced and she had no abnormal neurological signs. Her peripheral pulses were normal. Radiographs of the lumbar spine revealed generalised changes of disc space narrowing, osteophytes in the vertebral body. and arthritis of the posterior joints. A high-resolution CT scan demonstrated gross degenerative disease of the posterior joints with secondary narrowing of the spinal canal (Fig. 10). The scan may be repeated at various levels to define the stenotic segments before operation. A study is in progress to assess the place of this investigation in spinal stenosis either alone or in combination with water-soluble myelography (Fig. 11).

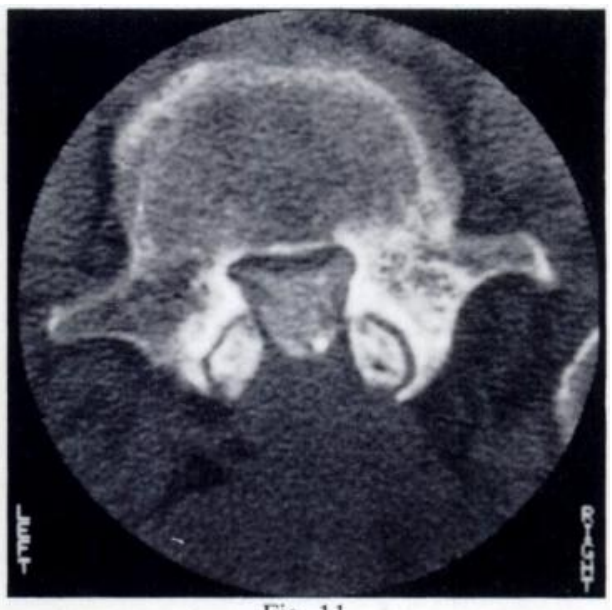

Fig. 11

Case 5. Spinal stenosis. Figure 10-Gross arthritic changes in the posterior joints. On the left the degenerate joint protrudes into the spinal canal. Figure 11-Scan with water-soluble myelography. The cauda equina is seen with nerve roots passing laterally in the direction of the intervertebral foramina. The posterior joints are arthritic.

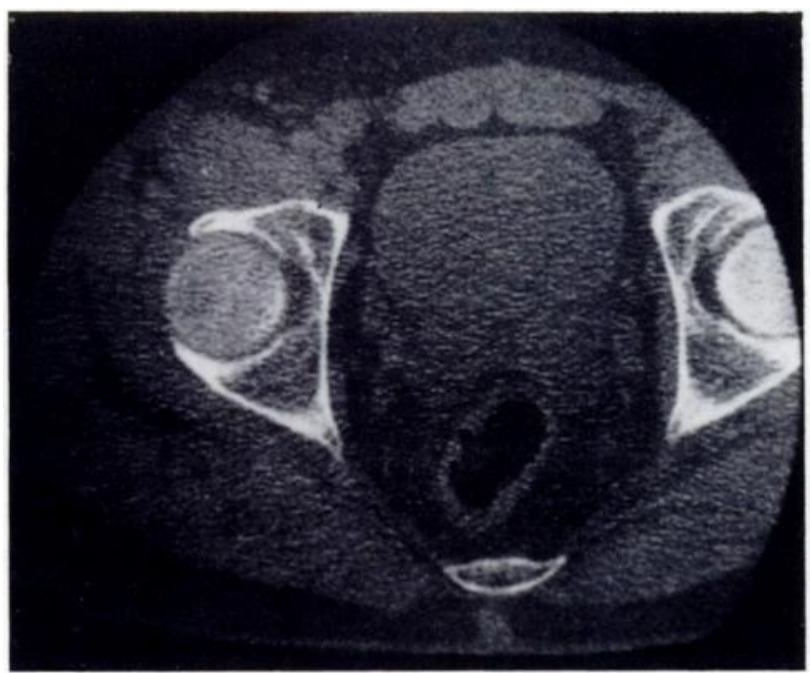

Fig. 12

Case 6. There is reduction in bone density about the right hip as compared to the left.
Case 6. Idiopathic "dystrophic" osteoporosis of the hip joint. A farmer aged 45 complained of pain in the right hip while walking which had progressed over six months. Examination revealed a slight antalgic limp and some pain at the extremes of a normal range of movement. Laboratory investigations including serology for rheumatoid factor, sedimentation rate, uric acid, and lupus erythematosus, were all normal. Radiographs were normal but careful scrutiny revealed a doubtful generalised osteoporosis around the right hip as compared with the left hip. A high-resolution CT scan revealed striking osteoporosis of the right femoral head and acetabulum as compared to the left side (Fig. 12). The patient was examined periodically and recovered spontaneously within the six months. The high-resolution scan proved more sensitive in demonstrating comparative osteoporosis and therefore allowed a more definite diagnosis.

Case 7. Osteoid osteoma of a vertebral body. A woman aged 28 complained for three years of dull pain in the right flank and front of the right thigh which was unrelated to activity and worse at night. Physical examination and laboratory studies were within normal limits. Radiographs of the spine revealed increased density and slight change of shape of the right side of the third lumbar vertebra (Fig. 13) but no definite lesion was seen on the lateral view (Fig. 14). Tomograms failed to demonstrate a nidus in the sclerotic area (Fig. 15). High-resolution CT scanning clearly demonstrated a small 


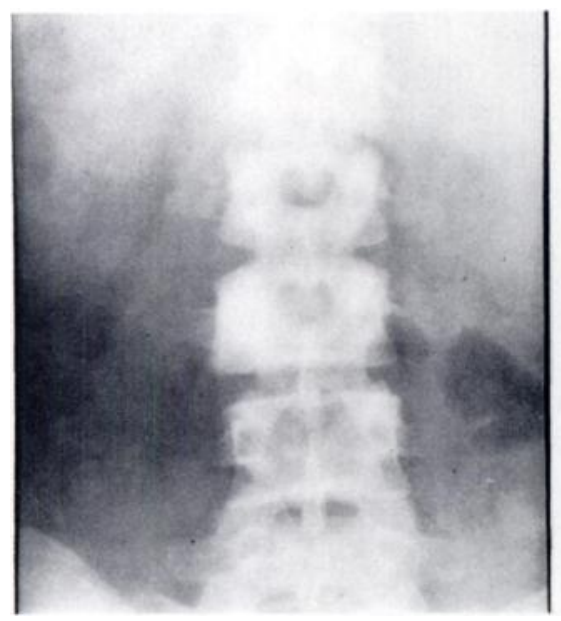

Fig. 13

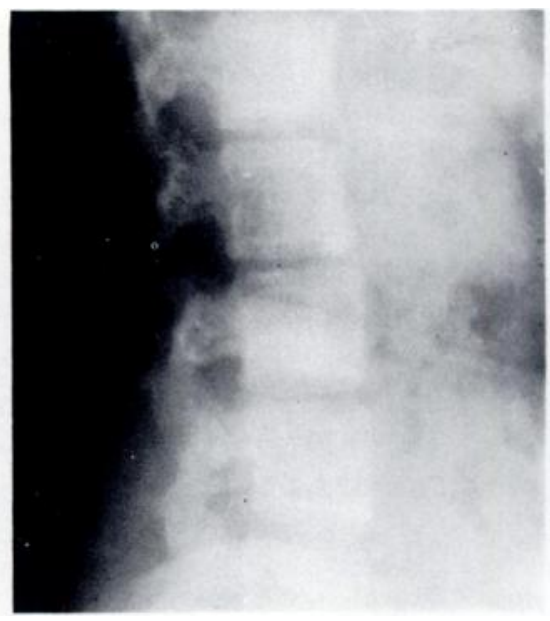

Fig. 14

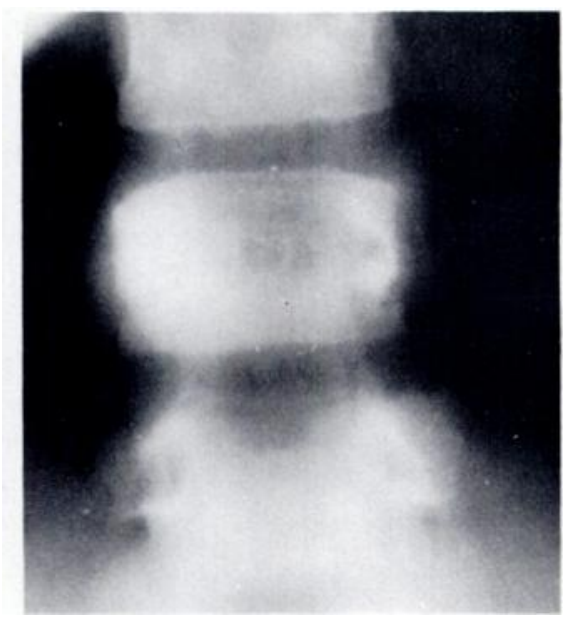

Fig. 15

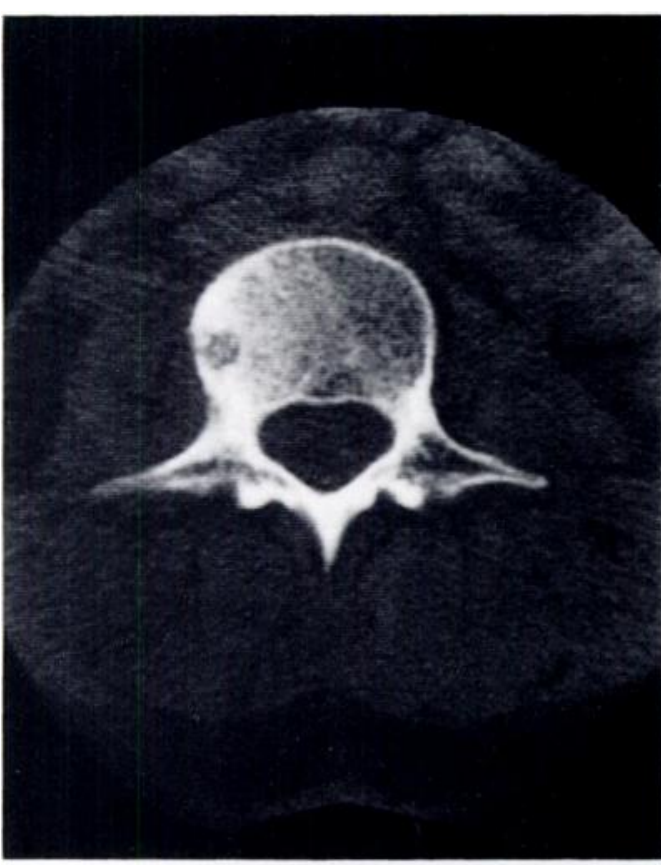

Fig. 16

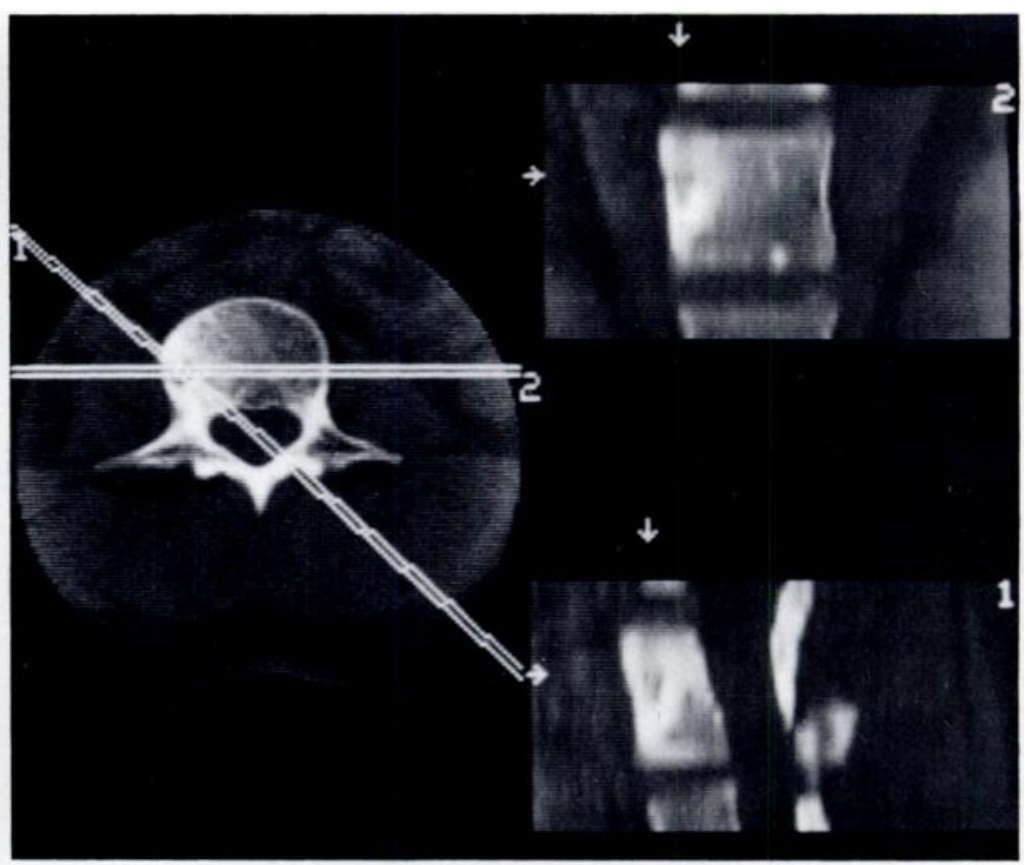

Fig. 17

Case 7. Osteoid osteoma of a vertebral body. Figures 13 and 14 -Conventional radiographs. Figure 15-Tomograph. Figure 16- High-resolution CT scan. Figure 17-Oblique sagittal and coronal films.

radiolucent nidus, surrounded by sclerotic bone, adjacent to the anterolateral cortical margin of the vertebra (Fig. 16). By correlation with oblique sagittal and coronal films (Fig. 17), a three-dimensional localisation of the nidus within the vertebral body could be obtained.

At operation, through a retroperitoneal approach, a twomillimetre opening in an area of roughened irregular cortex was noted under the periosteum at the site of the lesion seen on CT scan. A block excision of a 1.5-centimere cube was performed. Immediately after operation the patient had lost her pain and has remained free of pain one year later. This case shows the use of high-resolution CT scanning when conventional means have failed to provide exact localisation.

\section{DISCUSSION}

High-resolution computerised axial tomography is a non-invasive study with an acceptable dose of radiation. The image is not impaired by dense sclerotic bone, and the position of a lesion or nidus can be defined in three planes. In osteoid osteoma chronic pain may be present for months or years before a correct diagnosis is made, the nidus being usually a centimetre or less in diameter (Sherman 1947; Sankaran 1954; McLeod, Dahlin and Beabout 1976; Jackson, Reckling and Mantz 1977). Once it can be demonstrated, surgical excision can be recommended as a curative procedure. Accurate localisation of the nidus before operation facilitates the procedure and there is evidence that persistence of pain after operation is due to incomplete removal (Carroll 1953; Freiberger et al. 1959; Sim, Dahlin and Beabout 1975; Jackson et al. 1977; Ruge and Wiltse 1977; Norman 1978).

In plain radiographs of an osteoid osteoma in the cortex intense reactive bone formation may obscure the central nidus (Swee, McLeod and Beabout 1979). When subperiosteal or cancellous bone is involved, the reactive response is diminished and the radiographic 
diagnosis is more difficult (Ruge and Wiltse 1977; Swee et al. 1979). Conventional tomography may assist, but demands an exacting technique and sometimes fails to be diagnostic (Sankaran 1954; Caldicott 1969; Lisbona and Rosenthall 1979; Swee et al. 1979). Angiographic studies may give a false negative result and are unreliable (Worland, Ryder and Johnston 1975). Radionuclide imaging is of value in demonstrating the general vicinity of a reactive bone lesion and may be a better screening procedure than plain radiographs (Gore and Mueller 1975; Winter et al. 1977). A high-resolution CT scan is likely to provide the surgeon with more useful information than standard tomography or angiography as it reveals both the characteristic features and precise location of osteoid osteoma.

In our experience the high-resolution CT scanner is a useful adjunct in orthopaedic diagnosis whenever an axial view and the visualisation of details of fine bone structure are required (Case 3 ), when it is necessary to visualise very small lesions (Case 1) or to see details of bone structure within a plaster cast (Case 4). It is of great value when it is necessary to demonstrate a lesion in three-dimensional space, for example in planning an operation (Case 7).

We wish to thank Elscint Ltd, Haifa, for putting their high-resolution CT scanner at our disposal and for their technical advice.

\section{REFERENCES}

Berger PE, Kuhn JP. Computed tomography of tumors of the musculo-skeletal system in children. Radiology 1978;127:171-5. Caldicott WJH. Diagnosis of spinal osteoid osteoma. Radiology 1969:92:1192-5.

Carroll RE. Osteoid osteoma in the hand. J Bone Joint Surg [Am] 1953;35-A:888-93.

deSantos LA, Goldstein HM, Murray JA, Wallace S. Computed tomography in the evaluation of musculo-skeletal neoplasms. Radiology 1978:128:89-94.

Freiberger RH, Loitman BS, Helpern M, Thompeon TC. Osteoid osteoma: a report on 80 cases. AJR 1959:82:194.

Gore DR, Mueller HA. Osteoid-osteoma of the spine with localization aided by $99 \mathrm{mTc}$-polyphosphate bone scan: case report. Clin Orthop 1975;113:132-4.

Hammerschlag SB, Wolpert SM, Carter BL. Computed tomography of the spinal canal. Radiology 1976;121:361-7.

Hermann G, Rose JS. Computed tomography in bone and soft tissue pathology of the extremities. J Comput Assist Tomogr 1979;3:58-66.

Jackson RP, Reckling FW, Mantz FA. Osteoid osteoma and osteoblastoma: similar histologic lesions with different natural histories. Clin Orthop 1977:128:303-13.

Lee BCP, Kazam E, Newman AD. Computed tomography of the spine and spinal cord. Radiology 1978;128:95-102.

Lisbona R, Rosenthall L. Role of radionuclide imaging in osteoid osteoma. AJR 1979:132:77-80.

McLeod RA, Dahlin DC, Beabout JW. The spectrum of osteoblastoma. AJR 1976;126:321-35.

Norman A. Persistence or recurrence of pain: a sign of surgical failure in osteoid-osteoma. Clin Orthop 1978;130:263-6.

O'Connor JF, Cohen J. Computerized tomography (CAT scan, CT scan) in orthopaedic surgery.J Bone Joint Surg [Am] 1978;60-A:1096-8.

Ruge DA, Wiltse LL. Spinal disorders: diagnosis and treatment. Philadelphia: Lea \& Febiger. 1977:277.

Sankaran B. Osteoid osteoma. Surg Gynecol Obstet 1954;99:193-8.

Sherman MS. Osteoid osteoma: review of the literature and report of thirty cases. J Bone Joint Surg 1947:29:918-30.

Sim FH, Dahlin DC, Beabout JW. Osteoid osteoma: diagnostic problems. J Bone Joint Surg [Am] 1975:57-A:154-9.

Swee RG, McLeod RA, Beabout JW. Osteoid osteoma: detection, diagnosis and localization. Radiology 1979:130:117-23.

Wilson JS, Korobkin M, Genant HK, Bovill EG Jr. Computed tomography of musculoskeletal disorders. AJR 1978;131:55-61.

Winter PF, Johnson PM, Hilal SK, Feldman F. Scintigraphic detection of osteoid osteoma. Radiology 1977;122:177-8.

Worland RL, Ryder CT, Johnston AD. Recurrent osteoid osteoma: report of a case. J Bone Joint Surg [Am] 1975;57-A:277-8. 